\title{
Quantitative and qualitative evaluation on the accuracy of three intraoral scanners for human identification in forensic odontology
}

\author{
Eun-Jeong Bae ${ }^{1}$, Eun-Jin Woo ${ }^{2}$ \\ ${ }^{1}$ 3D Printer Technology Analysis Research Team, Cybermed Inc., Daejeon, ${ }^{2}$ Department of History, College of Liberal Art, Sejong University, Seoul, \\ Korea
}

\begin{abstract}
The purpose of this study was to analyze the accuracy of intra oral scanner (IOS) to confirm the applicability of IOS for the recording and analysis of tooth morphology in forensics. The less damaged mandible specimen with many teeth remaining was scanned three times using three types of intraoral scanners (CS3600, i500, and Trios3). For quantitative comparisons of the scanned images produced by these intraoral scanners, root mean square (RMS) values were computed using a three-dimensional analysis program and a one-way ANOVA was conducted with Tukey HSD (honestly significant difference) as a post-hoc analysis $(\alpha=0.05)$. The repeatability of the full scan data was highest with the i500 $(0.14 \pm 0.03 \mathrm{~mm})$, and the post-hoc analysis confirmed significant differences between the CS3600 and the i500 outcomes $(P$-value $=0.003)$. The repeatability of the partial scan data for the teeth in the mandible was highest with the $500(0.08 \pm 0.02 \mathrm{~mm})$, and the post-hoc analysis confirmed significant differences between the CS3600 and the i500 ( $P$-value $=0.016)$. The precision of the full scan data was highest with the i500 $(0.16 \pm 0.01 \mathrm{~mm})$ but the differences were not statistically significant $(P$-value $=0.091)$. Meanwhile, the precision of the partial scan data for the teeth in the mandible was highest with the Trios $3(0.22 \pm 0.02 \mathrm{~mm})$, but the differences were not statistically significant $(P$-value $=0.762)$. Considering that the scanning of other areas of the oral cavity in addition to the teeth is important in forensic odontology, the i500 scanner appears to be the most appropriate intraoral scanner for human identification. However, as the scope of oral scanning is generally limited to teeth in the practice of dentistry, additional discussions of how to apply the IOS in forensic odontology are needed. Ultimately, the results here can contribute to the overall discussion of the forensic applicability dental data produced by intraoral scanners.
\end{abstract}

Key words: Forensic odontology, Human identification, Intraoral scanner, Accuracy, Tooth

Received July 2, 2021; Revised August 30, 2021; Accepted September 3, 2021

\section{Introduction}

As the most durable parts of the human body, dental data enable the identification of unknown human remains, which

\section{Corresponding author:}

Eun-Jin Woo (iD

Department of History, College of Liberal Art, Sejong University, Seoul 05006, Korea

E-mail: redqin@sejong.ac.kr are useful for the reconstruction of a biological profile given that this data can allow one to ascertain age and sex in a forensic context $[1,2]$. Obviously, dental morphological traits help to restrict the number of identification candidates [3, 4]. Human identification through dental evidence is based on the detection of dental features by comparisons of antemortem (AM) dental records and post-mortem (PM) findings, and this process has been shown to be the preeminent method for human identification in mass disaster situations [5]. Typically, forensic radiologists evaluate the similarities and differences between AM and PM dental data based on 
radiographs [6].

Recent new technologies allow us to reconstruct an individual's dental traits with a high level of accuracy. As an example, intraoral scanners (IOSs) perform three-dimensional (3D) reconstructions of an individual's oral cavity and can analyze the adequacy of treatments [3]. Scanned 3D images of teeth and soft tissues in the oral cavity are considered alternatives to the traditional plaster model in dentistry [7]. In addition, scanned data can be permanently stored in a digital medium, such as a computer, and can be transmitted via the internet, enabling quick information exchanges [8]. For this reason, this technology enables more accurate and convenient diagnoses and treatments by those who practice dentistry [7]. Furthermore, the scope of its usage is currently expanding with the convergence of various technologies [8].

However, there are few studies that take advantage of these 3D dental images in a forensic context. For purposes of human identification, dental data produced by an intraoral scanner can be applied to population studies. The high reliability, ease of handling, and the reproducible 3D images produced through IOSs are appealing aspects for potential forensic applicability. For example, a study using 3D dental images obtained with IOSs exhibited a clear trend towards the validity of the use of $3 \mathrm{D}$ images for human identification purposes [3]. The study showed that intraoral 3D images present sufficient morphological detail to be classified for ancestry estimation. Furthermore, human identification using the intraoral digital scanning technique can be more rapid and convenient than the traditional method based on dental radiographs. Based on these advantages, 3D surface scanning images enable one to assess many opportunities during the presentation of case reports, for both teaching and research $[9,10]$. In this context, it is believed that future applications of such 3D data can be expected to contribute to advancing forensic odontology [11].

Although the field of digital scanning is expanding rapidly, it is necessary to consider the limitations of current
IOSs before the application of this technology, as all IOSs do not perform equally in terms of accuracy (i.e., trueness and precision) [12]. In forensic odontology, a fundamental question regarding the use of intraoral $3 \mathrm{D}$ images is whether they are capable of the recognition of dental morphological traits with a high level of accuracy. In this study, the accuracy of IOSs was quantitatively evaluated. The results of this study can be considered before the application of IOSs for the recording and analysis of teeth morphology and defects in forensic odontology.

\section{Materials and Methods}

The less damaged mandible specimen with many teeth remaining was selected to be scanned. The mandible was from an archaeological skeleton (estimated to be from the 16th-18th century) stored at the biological anthropology laboratory of the Department of Anthropology, Seoul National University (Fig. 1). This mandible is retrospective study data

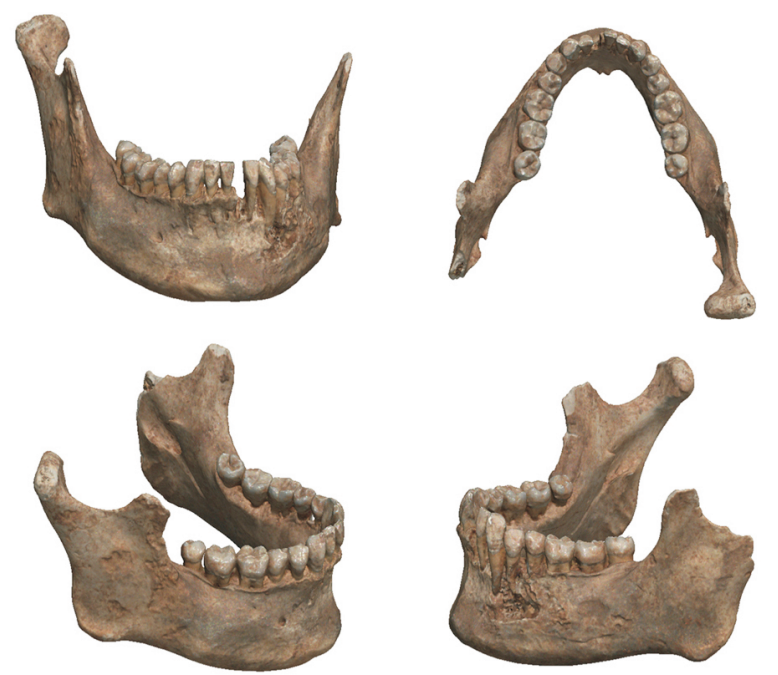

Fig. 1. The mandible used for scanning.

Table 1. Reference scanner (industrial) and intraoral scanner systems

\begin{tabular}{lll}
\hline \multicolumn{1}{c}{ Model } & \multicolumn{1}{c}{ Manufacturer } & Technical specifications \\
\hline Solutionix C500 & Medit, Seoul, Korea & $\begin{array}{c}\text { Light source-blue LED, 3D scanning principle-phase shifting optical } \\
\text { triangulation, camera resolusion, } 2 \times 5.0 \text { MP }\end{array}$ \\
CS3600 & Carestream, Rochester, NY, USA & Illumination-LED, amber, blue, green \\
i500 & Medit, Seoul, Korea & Color-3D full color streaming capture, imaging technology-3D \\
& & in motion video technology \\
Trios3 & 3Shape, Copenhagen, Denmark & Light source-LED, working principles-Ultrafast optical sectioning \\
\hline
\end{tabular}

LED, light-emitting diode; 3D, three dimensional. 
and is not subject to institutional review board review. The accuracy levels of three different scanners (CS3600; Carestream, Rochester, NY, USA; i500; Medit, Seoul, Korea, and Trios3; 3Shape, Copenhagen, Denmark) were evaluated and compared considering the features of the scanners, as shown in Table 1. These are the most popular scanners used in dentistry.

Scanning started with the occlusal surface of the left rearmost teeth. The teeth and roots were scanned in the occlusalbuccal-lingual order by an experienced researcher (first author) [13]. After scanning the teeth and roots, we attempted to scan as many areas as possible from the base to the head of the mandible.

The specimen was scanned ten times with each scanner to compare precision rates, and an industrial 3D scanner (C500, Medit) was used as the reference scanner to analyze the degree of repeatability. All scan data were stored in stereolithography format.

The root mean square (RMS) is used as a standard statistical metric to measure model performance outcomes [14]. We used the RMS value to analyze errors in the scan data. The RMS value was calculated using a 3D analysis program (Geomagic Control X inspection software; 3D Systems Inc., Cary, NC, USA).

First, in order to check the repeatability of each scanner, the data measured by the same scanner were compared to each other. The nesting method used was as follows. After the initial alignment of the two datasets to approximate the position, the best fit was selected by setting the sampling rate to $50 \%$. Thereafter, the sampling rate was set to $100 \%$ and a $3 \mathrm{D}$ comparison analysis was conducted. The analytical results were calculated for the minimum, maximum, mean, RMS, standard deviation, variance, plus average, minus average, and tolerance values.

The precision rates of three different scanners were analyzed based on one scan of the reference scan data, and the method used was identical to that described above. After repeatability and precision analyses of the full-scan data, only the section with teeth was extracted, and the repeatability and precision analyses were performed again. For teeth extraction, the Geomagic program was used to delete the teeth, leaving the anatomical cervical line.

To compare the repeatability and precision results, a oneway ANOVA analysis and a Tukey HSD (honestly significant difference) post hoc analysis were performed with a significance level of 0.05 (statistical software version 23.0; IBM Corp., Armonk, NY, USA).

Table 3. ANOVA analysis of precision of RMS values of full scan data and partial (teeth) scan data

\begin{tabular}{lcccccccc}
\hline \multirow{2}{*}{ Variable } & \multicolumn{3}{c}{ Full scan data } & & \multicolumn{3}{c}{ Partial scan data } \\
\cline { 2 - 3 } \cline { 6 - 7 } & $\begin{array}{c}\text { Mean } \\
\text { difference }\end{array}$ & $\begin{array}{c}\text { Standard } \\
\text { error }\end{array}$ & $P$-value & & $\begin{array}{c}\text { Mean } \\
\text { difference }\end{array}$ & $\begin{array}{c}\text { Standard } \\
\text { error }\end{array}$ & $P$-value \\
\hline C500 & & & & & & & \\
CS3600 & $-1.30^{*}$ & 0.22 & 0.002 & & $-0.11^{*}$ & 0.02 & 0.001 \\
i500 & -0.13 & 0.22 & 0.931 & & -0.04 & 0.02 & 0.259 \\
Trios & -0.68 & 0.22 & 0.063 & & $-0.09^{*}$ & 0.02 & 0.006 \\
CS3600 & & & & & & \\
i500 & $1.17^{*}$ & 0.22 & 0.003 & & $0.07^{*}$ & 0.02 & 0.016 \\
Trios & 0.62 & 0.22 & 0.088 & & 0.02 & 0.02 & 0.609 \\
i500 & & & & & & \\
Trios & -0.54 & 0.22 & 0.146 & & -0.05 & 0.02 & 0.091 \\
\hline
\end{tabular}

RMS, root mean square. One-way ANOVA test (post-hoc test: Tukey HSD). *Significant difference between groups $(P<0.05)$.

Table 2. Precision results of full scan data and partial (teeth) scan data

\begin{tabular}{|c|c|c|c|c|c|c|c|c|}
\hline \multirow{2}{*}{ Variable } & \multicolumn{4}{|c|}{ Full scan data } & \multicolumn{4}{|c|}{ Partial scan data } \\
\hline & C500 & CS3600 & i500 & Trios & C500 & CS3600 & i500 & Trios \\
\hline Min. & -0.10 & -6.35 & -0.61 & -4.77 & -0.51 & -0.81 & -0.63 & -0.85 \\
\hline Max. & 0.10 & 6.35 & 0.61 & 4.77 & 0.51 & 0.80 & 0.63 & 0.86 \\
\hline Mean & 0.00 & -0.22 & 0.01 & -0.03 & 0.00 & 0.00 & -0.01 & 0.01 \\
\hline RMS & 0.01 & 1.31 & 0.14 & 0.69 & 0.05 & 0.16 & 0.08 & 0.13 \\
\hline Standard deviation & 0.01 & 1.29 & 0.14 & 0.69 & 0.05 & 0.16 & 0.08 & 0.13 \\
\hline Var. & 0.00 & 1.83 & 0.02 & 0.48 & 0.00 & 0.02 & 0.01 & 0.02 \\
\hline+ Average & 0.01 & 0.37 & 0.12 & 0.24 & 0.02 & 0.11 & 0.05 & 0.09 \\
\hline -Average & -0.01 & -0.74 & -0.11 & -0.33 & -0.02 & -0.11 & -0.05 & -0.09 \\
\hline In Tol. (\%) & 98.93 & 23.47 & 30.57 & 26.64 & 93.45 & 33.64 & 71.29 & 47.26 \\
\hline Out Tol. (\%) & 1.07 & 76.53 & 69.43 & 73.36 & 6.55 & 66.36 & 28.71 & 52.74 \\
\hline Over Tol. (\%) & 0.55 & 40.28 & 32.50 & 34.30 & 3.57 & 31.69 & 15.93 & 22.56 \\
\hline Under Tol. (\%) & 0.51 & 36.25 & 36.93 & 39.06 & 2.98 & 34.67 & 12.78 & 30.18 \\
\hline
\end{tabular}

RMS, root mean square; Var, variance; In Tol. (\%), value within $50 \mu \mathrm{m}$; Out Tol. (\%), value excluding In Tol. (\%) from the entire data; Over Tol. (\%), value from 50-1,000 $\mu \mathrm{m}$; Under Tol. (\%), value from -50 to $-1,000 \mu \mathrm{m}$. 


\section{Results}

Tables 2 and 3 present the results of repeated scanning by the three scanners. In the full-scan data, repeatability was highest with the i500 device $(0.14 \pm 0.03 \mathrm{~mm})$, followed by the Trios3 $(0.69 \pm 0.11 \mathrm{~mm})$, and the CS3600 $(1.31 \pm 0.53 \mathrm{~mm})$. There were statistically significant differences among the groups, and a Tukey HSD post-hoc test confirmed a significant difference between the CS3600 and the i500 outcomes $(P$-value $=0.003)$.

In the partial-scan data, repeatability was highest with the i500 $(0.08 \pm 0.02 \mathrm{~mm})$, followed by the Trios $3(0.13 \pm 0.03$ $\mathrm{mm})$, and the CS3600 $(0.16 \pm 0.02 \mathrm{~mm})$. There were signifi-

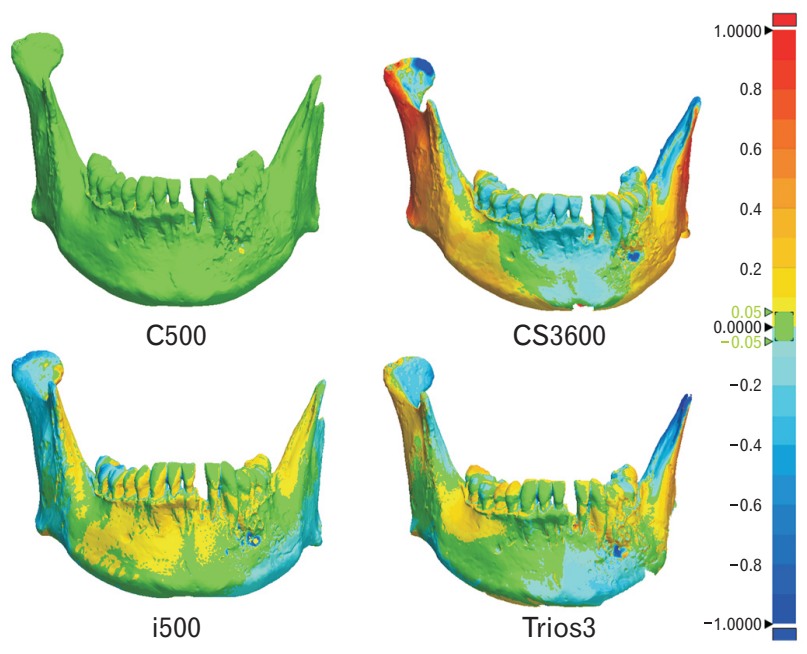

Fig. 2. Repeatability spectrum in the full scan data.

Table 4. Trueness results of full scan data and partial (teeth) scan data

\begin{tabular}{|c|c|c|c|c|c|c|}
\hline \multirow{2}{*}{ Variable } & \multicolumn{3}{|c|}{ Full scan data } & \multicolumn{3}{|c|}{ Partial scan data } \\
\hline & CS3600 & i500 & Trios & CS3600 & $\mathrm{i} 500$ & Trios \\
\hline Min. & -1.47 & -0.91 & -1.28 & -1.31 & -1.23 & -1.20 \\
\hline Max. & 1.47 & 0.91 & 1.28 & 1.31 & 1.23 & 1.19 \\
\hline Mean & 0.08 & 0.00 & -0.02 & -0.01 & -0.04 & -0.03 \\
\hline RMS & 0.33 & 0.16 & 0.22 & 0.24 & 0.23 & 0.22 \\
\hline $\begin{array}{l}\text { Standard } \\
\text { deviation }\end{array}$ & 0.31 & 0.16 & 0.22 & 0.24 & 0.23 & 0.22 \\
\hline Var. & 0.11 & 0.02 & 0.05 & 0.06 & 0.05 & 0.05 \\
\hline +Average & 0.26 & 0.09 & 0.12 & 0.13 & 0.09 & 0.09 \\
\hline -Average & -0.17 & -0.12 & -0.18 & -0.19 & -0.18 & -0.17 \\
\hline In Tol. (\%) & 20.20 & 36.86 & 27.77 & 27.63 & 44.66 & 38.60 \\
\hline Out Tol. (\%) & 79.80 & 63.14 & 72.23 & 72.37 & 55.34 & 61.40 \\
\hline Over Tol. (\%) & 29.73 & 28.01 & 35.14 & 31.80 & 29.17 & 30.85 \\
\hline Under Tol. (\%) & 50.07 & 35.13 & 37.09 & 40.57 & 26.16 & 30.56 \\
\hline
\end{tabular}

RMS, root mean square; Var, variance; In Tol. (\%), value within $50 \mu \mathrm{m}$; Out Tol. (\%), value excluding In Tol. (\%) from the entire data; Over Tol. (\%), value from $50-1,000 \mu \mathrm{m}$; Under Tol. (\%), value from -50 to $-1,000 \mu \mathrm{m}$. cant differences among the groups, and a post-hoc test again confirmed a significant difference between the CS3600 and the i500 outcomes $(P$-value $=0.016)$.

The amount of error for each instrument is represented as a spectrum in Fig. 2. Green indicates data within the tolerance threshold of $0.05 \mathrm{~mm}$, red indicates upper deviations, and blue lower deviations. There were fewer partial-scan data from the CS3600 compared to the reference data, while errors in the head of the mandible area were mostly in the upper deviation category.

In Fig. 3, only partial-scan data are compared. In the i500 data, some errors in the molar area were lower deviations, while the Trios 3 data showed both upper and lower deviations in the molar area. In the CS3600 data, both upper and lower deviations were apparent.

Tables 4 and 5 show the results of the precision RMS for the three scanners relative to the reference scanner result.

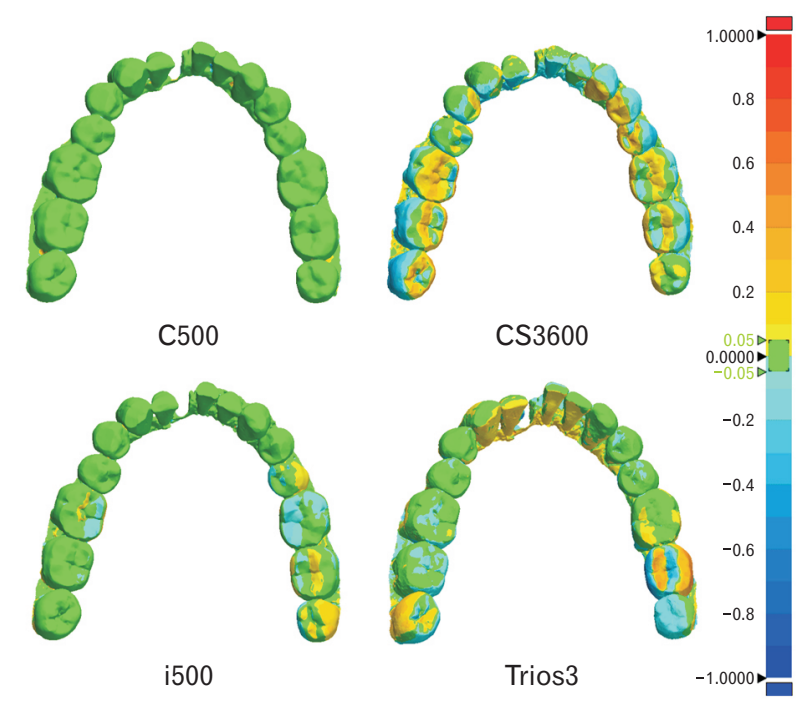

Fig. 3. Repeatability spectrum in the partial (teeth) scan data.

Table 5. ANOVA analysis of the precisions of the RMS values of full scan data and partial (teeth) scan data

\begin{tabular}{lccccc}
\hline \multicolumn{1}{c}{ Variable } & $\begin{array}{c}\text { Sum of } \\
\text { squares }\end{array}$ & $\begin{array}{c}\text { Degrees of } \\
\text { freedom }\end{array}$ & $\begin{array}{c}\text { Mean } \\
\text { square }\end{array}$ & F & $P$-value \\
\hline $\begin{array}{l}\text { Full scan data } \\
\text { Between-groups }\end{array}$ & 0.044 & 2 & 0.022 & 3.680 & 0.091 \\
$\quad$ Within-group & 0.035 & 27 & 0.006 & & \\
$\quad$ Total & 0.079 & 29 & & & \\
Partial scan data & & & & & \\
$\quad$ Between-groups & 0.000 & 2 & 0.000 & 0.285 & 0.762 \\
$\quad$ Within-group & 0.004 & 27 & 0.001 & & \\
Total & 0.004 & 29 & & & \\
\hline
\end{tabular}

RMS, root mean square. One-way ANOVA test. 


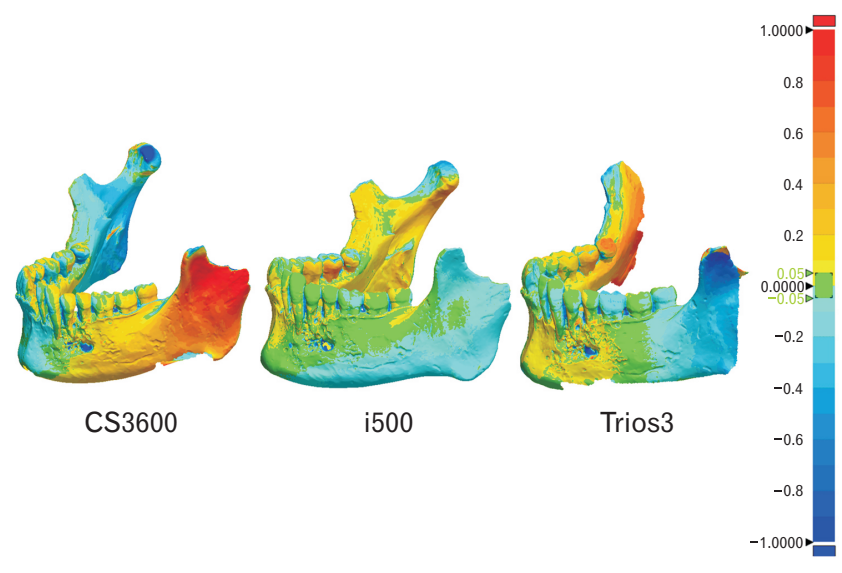

Fig. 4. Precision spectrum in the full scan data.

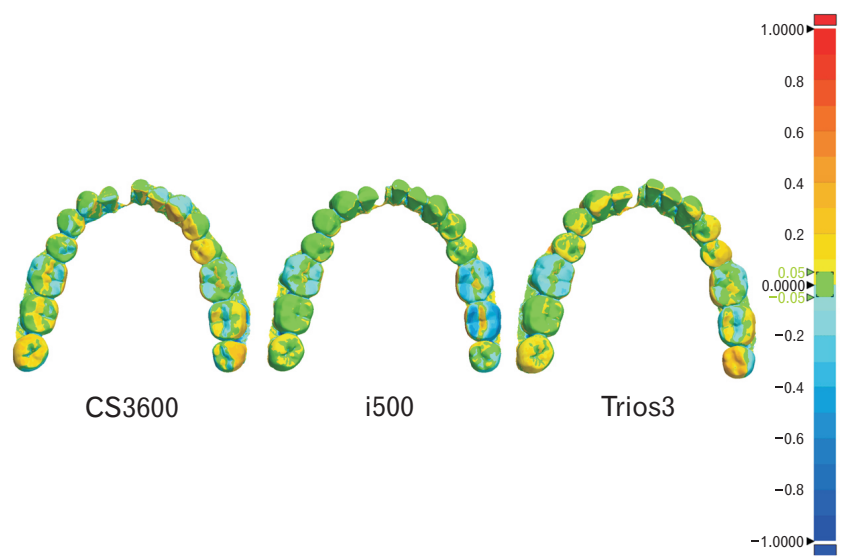

Fig. 5. Precision spectrum in the partial (teeth) scan data.

scanned a fully edentulous model and analyzed the precision and trueness. The precision of the fully edentulous model scan data was approximately $32-75 \mu \mathrm{m}$, higher than the results in this study. The trueness outcome was in the range of $60-106 \mu \mathrm{m}$, higher than the results in this study. Work by Lee et al. [17] found a trueness range of approximately 50-115 $\mu \mathrm{m}$ for the i500 and CS3600.

According to the American Dental Association, the clinically acceptable fit for indirect restorations is $50-100 \mu \mathrm{m}$, and several studies report clinical acceptable levels for restorations obtained from an IOS of $120 \mu \mathrm{m}[18,19]$. The results of this study showed a larger error value than the studies mentioned above. It is estimated that the mandible used in this study came from an individual who died during the 16 to 18 th century. As a result, over a long period of time, some of the mandible surface fractured or chipped. Also, some of the teeth may now be cracked and dry, and foreign matter may have prevented a surface smooth. Relevance can be gleaned from studies that show that cracks or debris on the tooth surface, or the complexity of the shape, can sometimes prevent a perfect scan or affect the accuracy [20]. Concerning this point, further research is needed to find the exact basis.

In this study, a larger area was scanned than is typically scanned for diagnosis or treatment in order to analyze a scan of the image across the mandible. Although the teeth were scanned well, some areas of the mandible could not be scanned well. In particular, scanning was difficult in sharp or angled areas such as coronoid process and mandibular angle. Mizumoto and Yilmaz [21] reported that sharp, undercut, angled, and steep structures are difficult to scan and result in inaccurate point clouds. Cuperus et al. [22] reported 
some omissions in the scan data because it is difficult to identify contact points when scanning the dental arch. The application of an IOS to forensic odontology requires additional research to ensure accurate scans of wider areas, which is a recent IOS-related issue. However, because we attempted to scan as much area as possible, we did not consider the time or amount of measurement data needed to obtain these scans.

In most IOS studies, the implications of forensic science are limited because laboratory or living patients are studied. In this study, real human remains (dried mandible) were used to check whether IOS can be applied to forensics. It may be difficult simply to contend that a dried mandible is directly related to forensic odontology, but discussing the applicability of an IOS, at least in the context of forensic odontology, can be expected to take the influence of dental identification to a new level in the realm of human identification.

The tooth shape scanned in 3D can be used in many ways. Further analysis can be carried out by modeling data obtained from scan images, or images can be $3 \mathrm{~d}$-printed and used for training after identification is completed. The accumulated images can also be used for artificial intelligence learning.

Traditional forensic analysis sometimes shows limitations related to the observer's subjective judgment [23, 24]. This research is expected to provide basic data pertaining to the progress of legal medicine toward digitalization and objective analysis. We also hope that the research results here will serve a scientific basis for improving the accuracy of PM data stored in 3D images.

In conclusion, there were differences in the precision and repeatability outcomes of full- and partial-scan data among the CS3600, i500, and Trios3 IOSs. For both full- and partial-scan data, the $\mathrm{i} 500$ had the highest precision. In terms of repeatability, the i500 was highest with full-scan data, while the Trios 3 had the highest repeatability with partial-scan data. Considering that scanning of other areas of the oral cavity besides the teeth is important in order for IOS systems to be used in forensic odontology, the i500 appears to be the most appropriate IOS. However, as the scope of oral scanning is generally limited to teeth in dental practice, further discussion is needed on how to apply IOSs in the field of forensic odontology.

Exploiting the set of benefits of IOSs for forensic dental identification would enable a quick, accurate and objective analysis. It is expected that the results of this study can be used as part of a scientific basis for improving the accuracy of PM data stored as $3 \mathrm{D}$ images.

\section{ORCID}

Eun-Jeong Bae: https://orcid.org/0000-0002-3098-7673

Eun-Jin Woo: https://orcid.org/0000-0002-7074-6022

\section{Author Contributions}

Conceptualization: EJB. Data acquisition: EJW. Data analysis or interpretation: EJB, EJW. Drafting of the manuscript: EJB. Critical revision of the manuscript: EJW. Approval of the final version of the manuscript: all authors.

\section{Conflicts of Interest}

No potential conflict of interest relevant to this article was reported.

\section{Acknowledgements}

The authors would like to thank Medit for the scanning of the mandible with the C500 device.

This work was supported by a grant from the National Research Foundation of Korea (NRF) funded by the Korean government (MSIT) (No. 2018R1A5A7023490). This work was also supported by a grant from the Daedeok Korea Innovation Cluster funded by the Korean government (MSIT) (No. 2021-DD-RD-0189).

\section{References}

1. Clark DH. An analysis of the value of forensic odontology in ten mass disasters. Int Dent J 1994;44:241-50.

2. Eboh DEO. Odontometric sex discrimination in young Urhobo adults of South-South Nigeria. Anat Cell Biol 2019;52:269-77.

3. Santo E, Pinho T, Teixeira A, Perez-Mongiovi D. Use of intraoral three-dimensional images for the identification of dental morphological traits related to ancestry estimation. J Forensic Sci Med 2021;7:70-3.

4. Kim WH, Nam SE, Park YS, Lee SP. Maxillary first molar wear: a longitudinal study of children. Anat Cell Biol 2018;51:251-9.

5. Clement JG. Odontology. In: Siegel JA, Saukko PJ, Houck MM, editors. Encyclopedia of Forensic Sciences. 2nd ed. Waltham: Elsevier/Academic Press; 2013. p.106-14.

6. Dedouit F, Savall F, Mokrane FZ, Rousseau H, Crubézy E, Rougé D, Telmon N. Virtual anthropology and forensic identi- 
fication using multidetector CT. Br J Radiol 2014;87:20130468.

7. Mangano F, Gandolfi A, Luongo G, Logozzo S. Intraoral scanners in dentistry: a review of the current literature. BMC Oral Health 2017;17:149.

8. Kravitz ND, Groth C, Jones PE, Graham JW, Redmond WR. Intraoral digital scanners. J Clin Orthod 2014;48:337-47.

9. Suese K. Progress in digital dentistry: the practical use of intraoral scanners. Dent Mater J 2020;39:52-6.

10. Lee SH, Nam SE, Lee SP. Evaluation of the effectiveness of the new tooth wear measurement parameters. Anat Cell Biol 2015;48:284-91.

11. Chomdej T, Pankaow W, Choychumroon S. Intelligent dental identification system (IDIS) in forensic medicine. Forensic Sci Int 2006;158:27-38.

12. Oh KC, Park JM, Moon HS. Effects of scanning strategy and scanner type on the accuracy of intraoral scans: a new approach for assessing the accuracy of scanned data. J Prosthodont 2020;29:518-23.

13. Nedelcu R, Olsson P, Nyström I, Rydén J, Thor A. Accuracy and precision of 3 intraoral scanners and accuracy of conventional impressions: a novel in vivo analysis method. J Dent 2018;69:110-8.

14. Chai T, Draxler RR. Root mean square error (RMSE) or mean absolute error (MAE)? - Arguments against avoiding RMSE in the literature. Geosci Model Dev 2014;7:1247-50.

15. Kim RJ, Park JM, Shim JS. Accuracy of 9 intraoral scanners for complete-arch image acquisition: a qualitative and quantitative evaluation. J Prosthet Dent 2018;120:895-903.e1.

16. Imburgia M, Logozzo S, Hauschild U, Veronesi G, Mangano
C, Mangano FG. Accuracy of four intraoral scanners in oral implantology: a comparative in vitro study. BMC Oral Health 2017;17:92.

17. Lee JH, Yun JH, Han JS, Yeo IL, Yoon HI. Repeatability of intraoral scanners for complete arch scan of partially edentulous dentitions: an in vitro study. J Clin Med 2019;8:1187.

18. McLean JW, von Fraunhofer JA. The estimation of cement film thickness by an in vivo technique. Br Dent J 1971;131:107-11.

19. Medina-Sotomayor P, Pascual-Moscardo A, Camps A I. Accuracy of 4 digital scanning systems on prepared teeth digitally isolated from a complete dental arch. J Prosthet Dent 2019;121:811-20.

20. Chen Y, Zhai Z, Li H, Yamada S, Matsuoka T, Ono S, Nakano T. Influence of liquid on the tooth surface on the accuracy of intraoral scanners: an in vitro study. J Prosthodont 2021 Apr 7 [Epub]. https://doi.org/10.1111/jopr.13358.

21. Mizumoto RM, Yilmaz B. Intraoral scan bodies in implant dentistry: a systematic review. J Prosthet Dent 2018;120:343-52.

22. Cuperus AM, Harms MC, Rangel FA, Bronkhorst EM, Schols JG, Breuning KH. Dental models made with an intraoral scanner: a validation study. Am J Orthod Dentofacial Orthop 2012;142:308-13.

23. Soomer H, Lincoln MJ, Ranta H, Penttilä A, Leibur E. Dentists' qualifications affect the accuracy of radiographic identification. J Forensic Sci 2003;48:1121-6.

24. Pinchi V, Norelli GA, Caputi F, Fassina G, Pradella F, Vincenti C. Dental identification by comparison of antemortem and postmortem dental radiographs: influence of operator qualifications and cognitive bias. Forensic Sci Int 2012;222:252-5. 